\title{
Comparative analysis and characterization of the gut microbiota of four farmed snakes from southern China
}

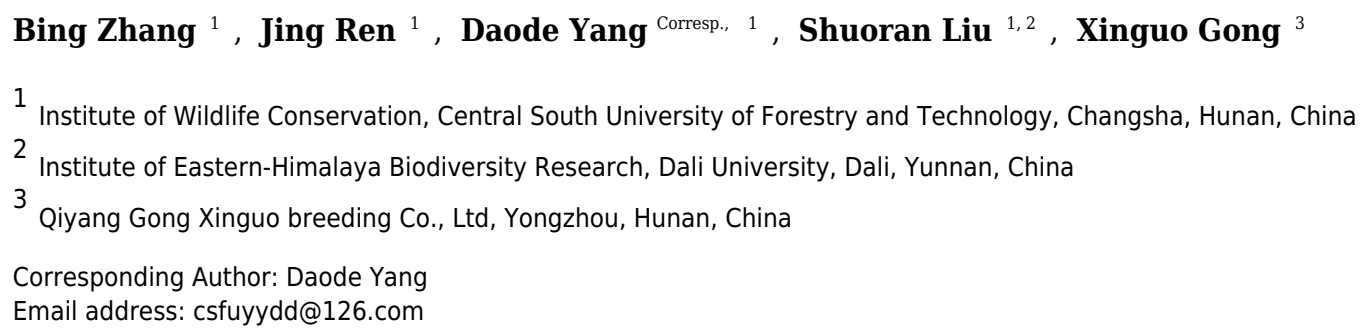

Background. The gut microbiota plays an important role in host immunity and metabolic homeostasis. Although analyses of gut microbiotas have been used to assess host health and foster disease prevention and treatment, no comparative comprehensive study, assessing gut microbiotas among several species of farmed snake, is yet available. In this study we characterized and compared the gut microbiotas of four species of farmed snakes (Naja atra, Ptyas mucosa, Elaphe carinata, and Deinagkistrodon acutus) using high-throughput sequencing of the 16S rDNA gene in southern China and tested whether there was a relationship between gut microbiotal composition and host species. Results. A total of 629 operational taxonomic units (OTUs) across 22 samples were detected. The five most abundant phyla were Bacteroidetes, Proteobacteria, Firmicutes, Fusobacteria, and Actinobacteria, while the five most abundant genera were Bacteroides, Cetobacterium, Clostridium, Plesiomonas, and Paeniclostridium. This was the first report of the dominance of Fusobacteria and Cetobacterium in the snake gut. Our phylogenetic analysis recovered a relatively close relationship between Fusobacteria and Bacteroidetes. Alpha diversity analysis indicated that species richness and diversity were highest in the gut microbiota of $D$. acutus and lowest in that of $E$. carinata. Significant differences in alpha diversity were detected among the four farmed snake species. The gut microbiotas of conspecifics were more similar to each other than to those of heterospecifics.

Conclusion. This study provides the first comparative study of gut microbiotas among several species of farmed snakes, and provides valuable data for the management of farmed snakes. In farmed snakes, host species affected the species composition and diversity of the gut microbiota. 


\section{Comparative analysis and characterization of the gut microbiota of}

2 four farmed snakes from southern China

3 Bing Zhang ${ }^{1}$, Jing Ren ${ }^{1}$, Daode Yang1*, Shuoran Liu' ${ }^{1,2}$ and Xinguo Gong ${ }^{3}$

$4{ }^{1}$ Institute of Wildlife Conservation, Central South University of Forestry and Technology, Changsha, Hunan, China

$5 \quad 2$ Institute of Eastern-Himalaya Biodiversity Research, Dali University, Dali, Yunnan, China

$6 \quad{ }^{3}$ Qiyang Gong Xinguo breeding Co., Ltd., Yongzhou, Hunan, China

7 Running head: Gut microbiota in farmed snakes

$8 *$ Contact information for the corresponding author: E-mail: csfuyydd@126.com

9 Brief information of the corresponding author: Prof. Daode Yang, from Central South University of Forestry

10 and Technology, Changsha, Hunan, China, with his research focusing on wildlife conservation and nature

11 reserve management. 
12 Background. The gut microbiota plays an important role in host immunity and metabolic homeostasis.

13 Although analyses of gut microbiotas have been used to assess host health and foster disease prevention and

treatment, no comparative comprehensive study, assessing gut microbiotas among several species of farmed

snake, is yet available. In this study we characterized and compared the gut microbiotas of four species of

farmed snakes (Naja atra, Ptyas mucosa, Elaphe carinata, and Deinagkistrodon acutus) using high-throughput

sequencing of the 16S rDNA gene in southern China and tested whether there was a relationship between gut

microbiotal composition and host species. Results. A total of 629 operational taxonomic units (OTUs) across

22 samples were detected. The five most abundant phyla were Bacteroidetes, Proteobacteria, Firmicutes,

Fusobacteria, and Actinobacteria, while the five most abundant genera were Bacteroides, Cetobacterium,

Clostridium, Plesiomonas, and Paeniclostridium. This was the first report of the dominance of Fusobacteria

and Cetobacterium in the snake gut. Our phylogenetic analysis recovered a relatively close relationship

between Fusobacteria and Bacteroidetes. Alpha diversity analysis indicated that species richness and diversity

were highest in the gut microbiota of D. acutus and lowest in that of E. carinata. Significant differences in

alpha diversity were detected among the four farmed snake species. The gut microbiotas of conspecifics were

more similar to each other than to those of heterospecifics. Conclusion. This study provides the first

comparative study of gut microbiotas among several species of farmed snakes, and provides valuable data for

the management of farmed snakes. In farmed snakes, host species affected the species composition and diversity of the gut microbiota.

\section{INTRODUCTION}

Vertebrates have evolved intimate symbiotic relationships with their internal microbes, especially those that 
fundamentally increased our understanding of evolution, health, disease, and aging (Kundu et al., 2017). Gut

microbiotas are extremely diverse, have unique functional characteristics, and may strongly affect the

physiological functions of the host (Costea et al., 2018). For example, the gut microbiota may regulate the immune response, thereby affecting energy homeostasis (Spiljar, Merkler \& Trajkovski, 2017) and nutrient metabolism (Shibata, Kunisawa \& Kiyono, 2017). Changes in the gut microbiota may influence the functions of the brain and nerves (Kundu et al., 2017). Therefore, the gut microbiota may be an important factor determining the growth, immunity, and survival rate of farmed animals (Hu et al., 2017; Rosshart et al., 2017).

The characterization of the gut microbiotas of farmed animals provides a scientific basis for disease diagnosis and health management (Kohl, Skopec \& Dearing, 2014; Jiang et al., 2017; Lyons et al., 2017). Such characterizations are also essential for the commercial production of economically important animals and the conservation management of endangered species (Larsen, Mohammed \& Arias, 2014).

Studies of gut microbiotas are primarily based on host fecal samples, as the collection of these samples is non-invasive. In mammals, fecal DNA reflects the composition and structure of the gut microbiota of the host (Ley et al., 2008; Costea et al., 2018). Previous studies indicate that mammal gut microbiotas are dominated by Firmicutes and Bacteroidetes (Ley et al., 2008; Hu et al., 2017). In birds, the microbiota demonstrates a similar phylum-level composition to that of mammals, being dominated by Bacteroidetes, Firmicutes, and Proteobacteria (Waite \& Taylor, 2014). In reptiles, the gut microbiota also appeared to be dominated by Firmicutes, followed by Bacteroidetes and Proteobacteria (Costello et al., 2010; Colston, Noonan \& Jackson, phylogenetic relationship among gut microbiota of the amniotes (reptiles, birds and mammals). A thorough 
54

55

56

57

consequently, our ability to manipulate the gut microbiota to treat disease (Kundu et al., 2017; Rosshart et al., 2017; Hu et al., 2017). However, there have been few studies of the gut microbiotas of snakes, an ancient group with more than 3,000 extant species (Uetz, Hošek \& Hallermann, 2016). Of the studies available, most investigated single species (Costello et al., 2010; Colston, Noonan \& Jackson, 2015; McLaughlin, Cochran \& Dowd, 2015; Shi \& Sun, 2017). Therefore, it remains necessary to comparatively assess the composition, diversity, and phylogeny of snake gut microbiotas.

In recent years, several snake species have been successfully artificially bred on a large scale; such artificialbreeding programs not only satisfy commercial needs, but also reduce pressure on wild snake populations to some extent (Hu et al., 2013; Hu, Tan \& Yang, 2013; Li, 2009). Naja atra (Elapidae), Ptyas mucosa (Colubridae), Elaphe carinata (Colubridae), and Deinagkistrodon acutus (Viperidae) are the snake species most commonly farmed in southern China ( $\mathrm{Li}$, 2009); N. atra and P. mucosa are listed in Appendix II of the Convention on International Trade in Endangered Species of Wild Fauna and Flora (CITES) (1990; https://www.cites.org/).

The aim of this study was to characterize the fecal microbiotas of four different species of farmed snakes in southern China, and to evaluate the effect of host species on the composition and diversity of the gut microbiota. This work serves as the first high-throughput sequencing analysis that compares the gut microbiotas of several farmed snake species. It is beneficial to study the gut microbiotas of snakes to improve the management of farmed snake populations.

\section{MATERIALS \& METHODS}

\section{Sample collection}

74 Fecal samples were collected from specimens of N. atra, P. mucosa, E. carinata, and D. acutus. All sampled 
75

76

77

78

79

80

81

82

83

84

85

86

87

88

89

90

91

92

93

94

95

snakes were healthy adults, hatched in 2014 and reared in similar farm environments. All snakes were kept in

farming rooms with a temperature of $28 \pm 2^{\circ} \mathrm{C}$, and a relative humidity of $80 \pm 5 \%$. Snakes were fed farmed

chicks (Gallus domestiaus) and mice (Mus musculus). All snakes were fed once a week, all given the same

food each feeding. For example, all snakes were fed chicks one time, and all snakes were fed mice the next

time. The fecal matter of each snake was sampled after they were fed the chicks. Fecal samples from N. atra,

D. acutus, and P. mucosa were collected at the Gong Xinguo snake farm, Yongzhou City, Hunan Province,

China from 8-11 July 2017; fecal samples from E. carinata were collected at the Lvdongshan snake farm,

Tujia-Miao Autonomous Prefecture of Xiangxi, Hunan Province, China on 26 August 2017. The wildlife

operation licenses of the two snake farms were authorized by the Forestry Department of Hunan Province. The

work was performed in accordance with the recommendations of the Institution of Animal Care and the Ethics

Committee of Central South University of Forestry and Technology (approval number: CSUFT NS \#

20175167). The fecal sampling procedures used in this study were non-invasive to the snakes.

Individual snakes were farmed in plastic rearing boxes. The boxes were numbered to allow us to distinguish individuals. Individual snakes used for sampling were randomly selected. Fresh fecal samples from same individuals were collected using a sterilized sampling spoon and put in the same centrifuge tube: N. atra (group 'Na'; n=6), P. mucosa (group 'Pmu'; n=4), E. carinata (group 'Ec'; n=6), and D. acutus (group 'Da';

$\mathrm{n}=6$ ). All fresh samples were immediately submerged in liquid nitrogen, and then frozen at $-20^{\circ} \mathrm{C}$ within $10 \mathrm{~h}$.

Samples were sent within $12 \mathrm{~h}$ on dry ice to the Wuhan Sample Center of Beijing Genomics Institute (BGI;

Wuhan, China) for DNA extraction.

\section{DNA extraction, sequencing}

Total DNA was extracted from the fecal samples using an E.Z.N.A. Stool DNA Kit (Omega Bio-tek, Inc., 
96 USA). The V4 hypervariable region of the 16S rDNA gene was amplified using polymerase chain reaction

97 (PCR), with the primers 515F (5'-GTGCCAGCMGCCGCGGTAA-3') and 806R (5'-

98 GGACTACHVGGGTWTCTAAT-3'). PCR products were purified with AmpureXP beads (Agencourt,

99 Beckman Coulter, California, USA) to remove any non-specific amplicons. Qualified libraries were pair-end

sequenced on a MiSeq System (Illumina, San Diego, CA, USA) with MiSeq reagents using the PE250

(PE251+8+8+251) sequencing strategy, following the manufacturer's instructions. All libraries were sequenced

on the Illumina MiSeq platform by the BGI (Wuhan, China).

103

104

105

106

107

108

109

110

111

112

\section{Bioinformatics and statistical analysis}

The raw sequencing data were filtered, and the low quality reads were removed using an in-house

procedure. The specific steps are as follows: 1) Sequence reads without an average quality of 20 over a $30 \mathrm{bp}$ sliding window based on the phred algorithm were truncated, and trimmed reads with less than $75 \%$ of their original length and their paired reads were removed; 2) Removal of reads contaminated by adapter (default parameter: 15 bases overlapped by reads and adapter with maximal 3 bases mismatch allowed); 3) Removal of reads with ambiguous basa ( $\mathrm{N}$ base), and its paired reads; 4) Removal of reads with low complexity (default: reads with 10 consecutive same base). The remaining high-quality reads were used for all subsequent analyses (Fadrosh et al., 2014). Paired end reads are merged to tags: If the two paired-end reads overlapped, the consensus sequence was generated by FLASH (Fast Length Adjustment of Short reads, v1.2.11), and the details of the method are as follows: 1) Minimal overlapping length: $15 \mathrm{bp}$; 2) Mismatching ratio of overlapped region: $<=0.1$. Removal of paired end reads without overlaps (Magoč \& Salzberg, 2011). Tags were aggregated into OTUs at 97\% similarity using USEARCH v7.0.1090 (Edgar, 2013). Species annotation was then performed on the OTUs by comparing the OTUs to the $16 \mathrm{~S}$ database 
117 (/RDP_set14/RDP_set14_NCBI_download_20151028) (Cole et al., 2013; Quast et al., 2012) with QIIME

118 v1.80 package (confidence threshold: 0.60; Caporaso et al., 2010).

119 The bacterial species corresponding to the recovered OTUs were identified by comparing the OTUs to the species database (/RDP_set14/RDP_set14_NCBI_download_20151028). Profiling area maps and histograms

for each sample set at the phylum, class, order, family, and genus levels were created. Heatmap analyses were also performed to compare bacterial community composition among the different host species. All bacterial classes with less than $0.5 \%$ relative abundance were combined into an "Others" class (Henderson et al., 2015 ;

Hu et al., 2017; Song et al., 2017).

The representative sequences were aligned against the Silva core set (Silva_108_core_aligned_seqs) using

PyNAST using 'align_seqs.py'. A representative OTU phylogenetic tree was constructed using the QIIME

(v1.80) built-in scripts including the fasttree method for tree construction (Caporaso et al., 2010). The most

abundant tags in each genus were chosen to represent the genus, and genus level phylogenetic tree was

(R Development Core Team 2014 [http://www.R-project.org/]) (Caporaso et al., 2010; Costello et al., 2010 ). 
138

139

140

141

142

143

144

145

146

147

148

149

150

151

152

153

154

155

156

158

157 Data quality evaluation

Alpha diversity was estimated by calculating the observed species index and the Shannon index using mothur v1.31.2 (http://www.mothur.org/wiki/Calculators). Difference analysis and mapping were performed in R

(v3.1.1) (White, Nagarajan \& Pop, 2009). To compare differences in bacterial diversity between pairs of snake species, beta diversity was analyzed based on Bray-Curtis dissimilarity using QIIME v1.80 (Caporaso et al., 2010).

The cladogram and biomarkers images were generated using linear discriminant analysis effect size (LEfSe) (Segata et al. 2011). The one-sample Kolmogorov-Smirnov (K-S) test was used to test the normality of the data. Then, we quantified the effect of host species on the five most abundant bacterial phyla using the general linear model (for the normally distributed data) or the generalized linear model (for the non-normally distributed data). A sequential Holm-Bonferroni correction was used to control for Type I error in SPSS v20.0 (IBM, Corp., Armonk, NY, USA). Differences in bacterial species abundance among samples were identified using the kruskal.test package (White, Nagarajan \& Pop, 2009) in R (v3.1.1), adjusting for the false discovery rate (FDR) and with the threshold P-value among groups set to 0.05 . Based on these results, the bacterial species that most influenced the differences in sample composition among groups were identified.

\section{Availability of supporting data}

The raw data obtained in this study have been deposited in National Centre for Biotechnology Information

(NCBI) Sequence Read Achieve (Bioproject: PRJNA516815; accession numbers:

SRR8494339-SRR8494360).

56 RESULTS

Across all samples, 727,310 sequences with an average length of $252 \mathrm{bp}$ were obtained (Table S1). The 
159

160

161

162

163

164

165

166

167

168

169

170

171

172

173

174

175

176

177

178

179

observed species and Shannon rarefaction curves tended to plateau, which showed that these sequence depths sufficiently captured the major microbiota in each sample (Fig. S1). Here, a total of 629 OTUs were obtained at the $97 \%$ sequence similarity cut-off levels and the number of OTUs shared by each sampling group was 109 (Table S1; Fig. S2). On average, $0.10 \%$ of all OTUs were unclassified at the phylum level (Fig. 1A), and $12.79 \%$ were unclassified at the genus level (Fig. 1B).

\section{Dominant bacterial taxa across all snake hosts}

The gut microbiotas of the four farmed snake species fell into 15 phyla, 18 classes, 22 orders, 35 families, and 58 genera (Table 1; Fig. 1; Fig. S3). In the overall dataset, the five most abundant phyla were identified as Bacteroidetes (30.98\%), Proteobacteria (24.80\%), Firmicutes (20.96\%), Fusobacteria (20.20\%), and Actinobacteria (1.53\%), while the five most abundant genera were Bacteroides (26.63\%), Cetobacterium (19.06\%), Clostridium (7.84\%), Plesiomonas (4.90\%), and Paeniclostridium (2.89\%) (Table S2). Phylogenetic analysis indicated that most genera fell into Bacteroidetes, Firmicutes, and Proteobacteria; only two genera fell into Fusobacteria (Fig. 2).

\section{Comparisons of gut microbiotas among the four snake species}

\section{(1) Alpha diversity analysis}

Alpha diversity indices (observed species, $P=0.001$; Shannon, $P=0.002$ ) differed significantly among the four snake species (Fig. 3A,B). For the community richness estimator (observed species index), each pairwise comparison among three species (D. acutus, E. carinata, and $N$. atra) was significant, while P. mucosa was not significantly different from E. carinata or N. atra. For the community diversity estimator (the Shannon index), among three species (D. acutus, E. carinata, and P. mucosa) was significant, but $N$. atra was not significantly different from E. carinata or P. mucosa (Fig. 3A,B). 
180

181

\section{(2) Similarity analysis}

The Bray-Curtis distance suggested that the bacterial community differences within each sample species were small; samples from the same species clustered together (with the exception of samples $\mathrm{Na} 4$ and $\mathrm{Na} 5$, which clustered with E. carinata; Fig. 3C). The PCA showed that the gut microbiotas from the same host species were more similar to each other than to the gut microbiotas from different host species, indicating that gut microbiotas were most similar within same snake species. Among the different snake species, E. carinata and $N$. atra were closest, indicating that the gut microbiotas of these two species were similar. In contrast, $D$. acutus was distantly separated from the other three species, indicating that the gut microbiota of D. acutus was dissimilar to those of the other three species (Fig. 3D).

Heatmap vertical clustering at the genus level showed that samples from the same snake species were tightly grouped on short branches, indicating that the composition and abundance of gut bacteria in the same sample were similar (with the exception of $\mathrm{Na} 2$ and Pmu3, which clustered with E. carinata; Fig. 4). These results were consistent with the beta diversity analysis.

\section{Differential microbes among species}

The LEfSe analysis was used to screen the differential microbes among species. The cladogram also showed 7 phyla, 11 classes, 17 orders, 29 families, and 45 genera were significantly enriched in distinct species (Fig. 5).

The GLM suggested that host species affected the relative abundances of Bacteroidetes, Firmicutes and Fusobacteria (with the exception of Proteobacteria), whereas the GLMs identified no significant effects of species on Actinobacteria abundance (Table 2). The relative abundances of the five most abundant genera across the four host species was shown in Fig. S4. D. acutus had a significantly higher abundances of genera Clostridium, Paeniclostridium, and Desulfovibrio. E. carinata had higher abundance of genera Edwardsiella, 
201 Escherichia, and Plesiomonas. Compared with other species, P. mucosa showed greater significantly in the

202 abundances of genera Cetobacterium.

\section{DISCUSSION}

204 Tens of billions of bacterial species have colonized vertebrates, typically in the gut (Ley et al., 2008; Costea et

al., 2018). The composition and structure of the normal gut microbiota can be used to assess animal health and

diagnose or prevent disease (Kundu et al., 2017; Rosshart et al., 2017; Hu et al., 2017). In the present study,

we provided the first comparative study of gut microbiotas among several species of farmed snakes in southern

China, and revealed the factor driving variation that will be useful for understanding the relationship between

gut microbiota and host species.

On average, we obtained 33,059 sequences per snake species (Table S1), consistent with previous similar

studies. For example, a mean of 33,690 sequences were obtained in the forest musk deer (Moschus berezovskii)

and the alpine musk deer (Moschus chrysogaster) (Hu et al., 2017); a mean of 30,000 sequences were obtained

in crocodile lizards (Shinisaurus crocodilurus) (Jiang et al., 2017); and a mean of 16,307 sequences were

obtained in black bears (Song et al., 2017). Thus, that the mean number of sequences and the depths of the

sequencing data for each individual sample we obtained were reasonable (Table S1; Fig. S1).

\section{Dominant gut microbes}

Bacteroidetes, Proteobacteria, Firmicutes, Fusobacteria, and Actinobacteria were the five most abundant phyla

in the gut microbiota of the four farmed snake species (Fig. 1A). This differed from mammals (Ley et al.,

2008), birds (Waite \& Taylor, 2014), and other reptiles (Colston, Noonan \& Jackson, 2015; Keenan, Engel \&

Elsey, 2013; McLaughlin, Cochran \& Dowd, 2015; Jiang et al., 2017). In previous studies of vertebrates, the 
222

223

224

225

226

227

228

229

230

231

232

233

234

235

236

237

238

239

240

241

242

physiological functions of the host with respect to metabolism and immunity (Thomas et al., 2011).

Lizards are another major taxon of reptiles ( 60\%) (Uetz, Hošek \& Hallermann, 2016). Previous reports

have indicated that the gut microbiota of lizards is dominated by the phyla Firmicutes (2.6-73\%), Bacteroidetes

(6.2-32.1\%), and Proteobacteria (19.1-56.4\%) (Hong et al., 2011; Ren et al., 2016; Jiang et al., 2017; Kohl et

al., 2017). Proteobacteria enrichment in the human gut was an indicator of gut microbiota imbalance and was

associated with host disease (Shin, Whon \& Bae, 2015). However, the proportion of Proteobacteria in the gut

microbiota of lizards was relatively high, although this proportion varied greatly by species. A similar situation

has been reported in snakes. For example, the gut microbiota of the Burmese python (Python bivittatus) was

10.1\% Proteobacteria (Costello et al., 2010), while that of the Timber rattlesnake (Crotalus horridus) was

85.0\% Proteobacteria (McLaughlin, Cochran \& Dowd, 2015). Similar results were also observed in the farmed

snake species analyzed here (16.4-36.9\%) (Table 2).

The proportion of Fusobacteria in the gut microbiotas of mammals, birds, and other snakes was relatively

small (Ley et al., 2008; Costello et al., 2010; Waite \& Taylor, 2014; Colston, Noonan \& Jackson, 2015;

McLaughlin, Cochran \& Dowd, 2015). However, Fusobacteria was a core gut microbiome of the American

alligator (Alligator mississippiensis), which could affect lumen biofilm development (Keenan, Engel \& Elsey,

2013). Here, Fusobacteria dominated the gut microbiotas of the farmed snakes; this is compositionally distinct

from other vertebrate gut microbiomes, including those of other reptiles, fish, birds, and mammals.

Bacteroides and Cetobacterium were the dominant bacterial genera in gut microbiota of the farmed snakes

(Fig. 2). Bacteroides maintain a complex and beneficial relationship in the host gut, and the symbiotic

relationships between these bacteria and their hosts have been widely studied (Thomas et al., 2011). For

example, Bacteroides species have complex systems for sensing nutrient utilization, regulating nutrient 
243

244

245

246

247

248

249

250

251

252

253

254

255

256

257

258

259

260

261

262

263

metabolism, and acquiring and hydrolyzing otherwise indigestible dietary polysaccharides (Xu et al., 2003).

Bacteroides species control host gut homeostasis by interacting with the host immune system (Wexler, 2007).

Here, the gut microbiotas of the farmed snakes were dominated by Bacteroides, especially the samples from $E$.

carinata (42.09\%) and N. atra (40.17\%) (Fig. 3), indicating that the gut microbiota in snakes are species dependent. All Cetobacterium species are obligate anaerobes in phylum Fusobacteria (Fig. 2). Cetobacterium

was the dominant genus in the gut microbiotas of all the farmed snakes analyzed herein; this is the first report of the dominance of this genus in the gut microbiotas of snakes.

\section{Fusobacteria in gut microbiotas of farmed snakes}

Fusobacteria is a little-studied bacterial phylum, with a somewhat uncertain phylogenetic position (Keenan,

Engel \& Elsey, 2013). The results of the present study indicated that only two genera fell into Fusobacteria by phylogenetic analysis, Cetobacterium and Fusobacterium (Fig. 2). However, it is possible that Fusobacteria includes additional unclassified genera, and/or that the Fusobacteria have been undersampled in previous studies of gut microbiotas (Keenan, Engel \& Elsey, 2013). Previous studies have suggested that Fusobacteria have a core genome dissimilar to that of other bacterial lineages (Mira et al., 2004). Phylogenetic and comparative genomics analyses indicate that this phylum is closely affiliated with Bacteroidetes and Firmicutes, and may be derived from the Firmicutes (Mira et al., 2004). Phylogenetic analysis recovered a close relationship between Fusobacteria and Bacteroidetes, indicating a relatively close evolutionary relationship (Fig. 2). Bacteroidetes is one of the major lineages of bacteria, arising early in bacterial evolution

(Wexler, 2007). Therefore, the evolutionary relationship between Fusobacteria and Bacteroidetes should be further investigated.

Fusobacteria species play a critical role in initial biofilm development (Mira et al., 2004), suggesting that 
264

265

266

267

268

269

270

271

272

273

the presence of these species in the guts of the farmed snakes may affect the development of the lumen membrane (Keenan, Engel \& Elsey, 2013). Cetobacterium was first isolated from the intestinal contents of a porpoise and from the mouth lesion of a minke whale (Balaenoptera acutorostrata) (Foster et al., 1995). Species in this genus transform peptones and carbohydrate into acetic acid (Edwards, Logan \& Gharbia, 2015). Because Fusobacteria and Cetobacterium dominated the gut microbiotas of the farmed snakes, species in these taxa were likely commensal inhabitants of snake guts. It is therefore possible to speculate that, in snakes, Fusobacteria and Cetobacterium play important roles in digestive organ development and in nutritional metabolism.

\section{The relationship between gut microbiota and host species}

Many factors affect the vertebrate gut microbiotas, including host species, diet, and age (Ley et al., 2008;

Waite \& Taylor, 2014; Hu et al., 2017; Jiang et al., 2017). The gut microbiota may also vary in different regions of the gut tract (Ley et al., 2008; Waite \& Taylor, 2014). Diet and host species influence the composition of the gut microbiota more than other factors (Waite \& Taylor, 2014). The gut microbiota of the Burmese python was dominated by Firmicutes and Bacteroidetes (Costello et al., 2010), while the gut microbiota of the timber rattlesnake was uniquely dominated by Proteobacteria (McLaughlin, Cochran \& Dowd, 2015). Bacteroidetes, Firmicutes, and Proteobacteria also dominated the gut microbiota of the cottonmouth snake (Colston, Noonan \& Jackson, 2015). Therefore, the dominant bacterial phyla vary based on snake species. However, diet, age, habitat, and research method varied in previous studies of snake microbiotas, which possibly affected the distribution of bacterial species abundance at the phylum level. Here, the composition of gut microbiota was unique to each species of farmed snake. The four species shared similar breeding modes, but the composition and diversity of the gut microbiota were more similar within species and 
285

286

287

288

289

290

291

292

293

294

295

296

297

298

299

300

301

302

303

304

305

more different between species. This, suggested a relationship between the composition and diversity of the gut microbiota and the host species. However, the fecal samples of $E$. carinata originated from a different farm from the other three, which may have had an impact on the study results. The composition and diversity of the E. carinata gut microbiota differed from those of the other three species. For example, the community richness estimate for the fecal samples of E. carinata was significantly lower than that of N. atra and D. acutus (Fig.

3A). E. carinata had higher abundances of the dominant genera Edwardsiella, Escherichia, and Plesiomonas

(Fig. S4). We therefore hypothesize that variations among farms may also be a factor contributing to the composition and community structures of host gut microbiotas. However, the community diversity estimate for the fecal samples of E. carinata did not differ significantly from N. atra (Fig. 3B). Bray-Curtis distance, PCA, and Heatmap vertical clustering showed that the gut microbiotas of E. carinata and $N$. atra were somewhat similar. Therefore, farm variation may not be an important factor altering the gut microbiotas of farmed snakes. In addition, the species studied here were similar with respect to diet, health, farmed environment, and age. This suggested that host species was probably the important factor shaping the microbiot.

\section{CONCLUSION}

The compositions of the gut microbiotas of four farmed snake species in southern China were different to those of other snakes and vertebrates. The gut bacteria of these four species fell into 15 phyla, 18 classes, 22 orders, 35 families, and 58 genera. The five most abundant phyla were Bacteroidetes, Proteobacteria, Firmicutes, Fusobacteria, and Actinobacteria, while the five most abundant genera were Bacteroides, Cetobacterium, Clostridium, Plesiomonas, and Paeniclostridium. This was the first report that Fusobacteria and Cetobacterium dominated the gut microbiotas of snake species. Gut microbiotal diversity was highest in $D$. acutus and lowest in E. carinata. There were interspecific differences in gut microbiota composition and diversity among the four 
306

307

308

309

310

311

312

313

314

315

316

317

318

319

320

321

322

323

324

325

326

farmed snake species. Our results supported our hypothesis that host species was an important factor affecting the gut microbiotas of snakes. Further studies of snake gut microbiotas should investigate the relationship between phylogenetic position and function, as well as the characteristics of dominant bacteria that were unclassifiable. It is important to determine whether the immunity and growth of farmed snake populations can be improved by inoculating fecal suspensions generated by healthy wild snakes into the guts of farmed conspecifics.

\section{REFERENCES}

Caporaso JG, Kuczynski J, Stombaugh J, Bittinger K, Bushman FD, Costello EK, Fierer N, Peña AG, Julia K Goodrich, Gordon JI, Huttley GA, Kelley ST, Knights D, Koenig JE, Ley RE, Lozupone1 CA, McDonald1 D, Muegge BD, Pirrung1 M, Reeder1 J, Sevinsky JR, Turnbaugh PJ, Walters WA, Widmann1 J, Yatsunenko T, Zaneveld J, Knight R. 2010. QIIME allows analysis of high-throughput community sequencing data. Nature Methods 7, 335-336 DOI 10.1038/nmeth.f.303.

Chen H, Boutros PC. 2011. Venndiagram: a package for the generation of highly-customizable venn and euler diagrams in r. Bmc Bioinformatics 12, 35 DOI 10.1186/1471-2105-12-35.

Cole JR, Wang Q, Fish JA, Chai B, McGarrell DM, Sun Y, Brown CT, Porras-Alfaro A, Kuske CR,Tiedje JM. 2013. Ribosomal Database Project: data and tools for high throughput rRNA analysis. Nucleic Acids Research 42, D633-D642 DOI 10.1093/nar/gkt1244.

Colston TJ, Noonan BP, Jackson CR. 2015. Phylogenetic analysis of bacterial communities in different regions of the gastrointestinal tract of Agkistrodon piscivorus, the cottonmouth snake. PloS One 10, e0128793 DOI 10.1371/journal.pone.0128793. 
327

328

329

330

331

332

333

334

335

336

337

338

339

340

341

342

343

344

345

346

347
M, Huttenhower C, Jeffery IB, Knights D, Lewis JD, Ley RE, Ochman H, O'Toole PW, Quince C, Relman DA, Shanahan F, Sunagawa S, Wang J, Weinstock GM, Wu GD, Zeller G, Zhao L, Raes J, Knight R, Bork P. 2018. Enterotypes in the landscape of gut microbial community composition. Nature Microbiology 3, 8-16 DOI 10.1038/s41564017-0072-8.

Costello EK, Gordon JI, Secor SM, Knight R. 2010. Postprandial remodeling of the gut microbiota in Burmese pythons. ISME Journal 4, 1375-1385 DOI 10.1038/ismej.2010.71.

Dray S, Dufour A. 2007. The ade4 package: implementing the duality diagram for ecologists. Journal of Statistical Software 22, 1-20 DOI 10.18637/jss.v022.i04.

Edgar RC. 2013. UPARSE: highly accurate OTU sequences from microbial amplicon reads. Nature Methods 10, 996-998 DOI 10.1038/nmeth.2604.

Edwards KJ, Logan JMJ, Gharbia SE. 2015. Cetobacterium. Bergey's Manual of Systematics of Archaea and Bacteria. John Wiley and Sons, Ltd DOI 10.1002/9781118960608.gbm00767.

Fadrosh DW, Ma B, Gajer P, Sengamalay N, Ott S, Brotman RM, Ravel J. 2014. An improved dual-indexing approach for multiplexed 16S rRNA gene sequencing on the Illumina MiSeq platform. Microbiome 2, 1-7 DOI 10.1186/2049-2618-2-6.

Foster G, Ross HM, Naylor RD, Collins MD, Ramos CP, Garayzabal FF, Reid RJ. 1995. Cetobacterium ceti gen. nov. sp. nov. a new gram-negative obligate anaerobe from sea mammals. Letters in Applied Microbiolory 21, 202-206 DOI 10.1111/j.1472-765X.1995.tb01041.x.

Gao QX, Wu TX, Wang JB. 2010. Advance in research on symbiotic relationship between intestinal bacterial and their Host. Chinese Journal of Animal Nutrition 22, 519-526 DOI 10.3969/j.issn.1006-267x.2010.03.002.

Henderson G, Cox F, Ganesh S, Jonker A, Young W, Collaborators GRC, Janssen PH. 2015. Rumen microbial community composition varies with diet and host, but a core microbiome is found across a wide geographical range. Scientific Reports $\mathbf{5}$, 
14567 DOI $10.1038 /$ srep14567.

Hong PY, Wheeler E, Cann IK, Mackie RI. 2011. Phylogenetic analysis of the fecal microbial community in herbivorous land and marine iguanas of the galápagos islands using 16s rrna-based pyrosequencing. Isme Journal 5, 1461 DOI 10.1038/ismej.2011.33.

Hu L, Geng S, Li Y, Cheng S, Fu X, Yue X, Han X. 2017. Exogenous fecal microbiota transplantation from local adult pigs to crossbred newborn piglets. Frontiers in Microbiology 8, 2663 DOI 10.3389/fmicb.2017.02663.

Hu MX, Tan QY, Li Y, Yang DD. 2013. Allopatric captive rearing in the tropics increases the growth Rates of Deinagkistrodon acutus snakelets. Scientia Silvae Sinicae 49, 194-198 DOI 10.11707/j.1001-7488.20130526.

Hu MX, Tan QY, Li Y, Yang DD. 2013. Relationships among female body size, clutch size, and egg size in captive Deinagkistrodon acutus. Acta Ecologica Sinica 33, 1778-1783 DOI 10.5846/stxb201202130186.

Hu X, Liu G, Shafer ABA, Wei Y, Zhou J, Lin S, Wu H, Zhou M, Hu D, Liu S. 2017. Comparative analysis of the gut microbial communities in forest and alpine musk deer using high-throughput sequencing. Front Microbiol 8, 572 DOI 10.3389/fmicb.2017.00572.

Jiang HY, Ma JE, Li J, Zhang XJ, Li LM, He N, Liu HY, Luo SY, Wu ZJ, Han RC,Chen JP. 2017. Diets alter the gut microbiome of crocodile lizards. Frontiers in Microbiology 8, 2073 DOI 10.3389/fmicb.2017.02073.

Keenan SW, Engel AS, Elsey RM. 2013. The alligator gut microbiome and implications for archosaur symbioses. Scientific Reports 3, 2877 DOI 10.1038/srep02877.

Kohl KD, Brun A, Magallanes M, Brinkerhoff J, Laspiur A, Acosta JC, Laviedes-vidal E, Bordenstein SR. 2017. Gut microbial ecology of lizards: insights into diversity in the wild, effects of captivity, variation across gut regions and transmission. Molecular Ecology 26, 1175-1189 DOI 10.1111/mec.13921.

Kohl KD, Skopec MM, Dearing MD. 2014. Captivity results in disparate loss of gut microbial diversity in closely related hosts. 
Conservation Physiology 2, cou009 DOI 10.1093/conphys/cou009.

Kundu P, Blacher E, Elinav E, Pettersson S. 2017. Our gut microbiome: the evolving inner self. Cell 171, 1481-1493 DOI 10.1016/j.cell.2017.11.024.

Larsen AM, Mohammed HH, Arias CR. 2014. Characterization of the gut microbiota of three commercially valuable warmwater fish species. Journal of Applied Microbiology 116, 1396-1404 DOI 10.1111/jam.12475.

Ley RE, Hamady M, Lozupone C, Turnbaugh PJ, Ramey RR, Bircher JS, Schlegel ML, Tucker TA, Schrenzel MD, Knight R, Gordon JI. 2008. Evolution of mammals and their gut microbes. Science 320, 1647-1651 DOI 10.1126/science.1155725.

Ley RE, Lozupone CA, Hamady M, Knight R, Gordon JI. 2008. Worlds within worlds: evolution of the vertebrate gut microbiota. Nature Reviews Microbiology 6, 776-788 DOI 10.1038/nrmicro1978.

Li M, Wang BH, Zhang MH, Rantalainen M, Wang SY, Zhou HK, Zhang Y, Shen J, Pang XY, Zhang ML, Wei H, Chen Y, Lu HF, Zuo J, Su MM, Qiu YP, Jia W, Xiao CN, Smith LM, Yang SL, Holmes E, Tang HR, Zhao GP, Nicholson JK, Li LJ, Zhao LP. 2008. Symbiotic gut microbes modulate human metabolic phenotypes. Proceedings of the National Academy of Sciences of the United States of America, 105(6), 2117-2122 DOI 10.1073/pnas.0712038105.

Li PP. 2009. Status of conservation and farmed breeding of snakes in China. Journal of Snake 21, 173-176 DOI 10.3969/j.issn.1001-5639.2009.03.001.

Lyons PP, Turnbull JF, Dawson KA, Crumlish M. 2017. Phylogenetic and functional characterization of the distal intestinal microbiome of rainbow trout oncorhynchus mykiss from both farm and aquarium settings. Journal of Applied Microbiology 122, 347 DOI 10.1111/jam.13347.

Magoč T, Salzberg SL. 2011. FLASH: fast length adjustment of short reads to improve genome assemblies. Bioinformatics 27, 2957-2963 DOI 10.1093/bioinformatics/btr507.

Mallick H, Ma S, Franzosa EA, Vatanen T, Morgan XC, Huttenhower C. 2017. Experimental design and quantitative analysis 
of microbial community multiomics. Genome Biology 18, 228 DOI 10.1186/s13059-017-1359-z.

McLaughlin RW, Cochran PA, Dowd SE. 2015. Metagenomic analysis of the gut microbiota of the Timber rattlesnake, Crotalus horridus. Molecular Biology Reports 42, 1187-1195 DOI 10.1007/s11033-015-3854-1.

Mira A, Pushker R, Legault BA, Moreira D, Rodríguezvalera F. 2004. Evolutionary relationships of fusobacterium nucleatum based on phylogenetic analysis and comparative genomics. Bmc Evolutionary Biology 4, 50 DOI 10.1186/1471-2148-4-50.

Quast C, Pruesse E, Yilmaz P, Gerken J, Schweer T, Yarza P, Peplies J, Glöckner FO. 2012. The SILVA ribosomal RNA gene database project: improved data processing and web-based tools. Nucleic Acids Research 41, D590-D596 DOI 10.1093/nar/gks1219.

Ren T, Kahrl AF, Wu M, Cox RM. 2016. Does adaptive radiation of a host lineage promote ecological diversity of its bacterial communities? a test using gut microbiota of anolis lizards. Molecular Ecology 25, 4793-4804 DOI 10.1111/mec.13796.

Rosshart SP, Vassallo BG, Angeletti D, Hutchinson DS, Morgan AP, Takeda K, Hickman HD, McCulloch JA, Badger JH, Ajami NJ, Trinchieri G, Pardo-Manuel de Villena F, Yewdell JW, Rehermann B. 2017. Wild mouse gut microbiota promotes host fitness and improves disease resistance. Cell 171(5), 1015-1028 DOI 10.1016/j.cell.2017.09.016.

Schloss PD, Westcott SL, Ryabin T, Hall JR, Hartmann M, Hollister EB, Lesniewski RA, Oakley BB, Parks DH, Robinson CJ, Sahl JW, Stres B, Thallinger GG, Horn DJV, Webe CF. 2009. Introducing mothur: open-source, platform-independent, community-supported software for describing and comparing microbial communities. Applied and Environmental Microbiology 75, 7537-7541 DOI 10.1128/AEM.01541-09.

Segata N, Izard J, Waldron L, Gevers D, Miropolsky L, Garrett WS, Huttenhower C. 2011. Metagenomic biomarker discovery and explanation. Genome Biol 12, R60 DOI 10.1186/gb-2011-12-6-r60.

Shi YD, Sun H. 2017. Characterization of the intestinal microflora of Elaphe taeniura. Journal of Hunan Agricultural University (Natural Sciences) 43, 292-297 DOI 10.13331/j.cnki.jhau.2017.03.013. 
411 Shibata N, Kunisawa J, Kiyono H. 2017. Dietary and microbial metabolites in the regulation of host immunity. Frontiers in Microbiology 8, 2171 DOI 10.3389/fmicb.2017.02171.

413

Shi NR, Whon TW, Bae JW. 2015. Proteobacteria: microbial signature of dysbiosis in gut microbiota. Trends in Biotechnology 33, 496-503 DOI 10.1016/j.tibtech.2015.06.011.

Song C, Wang B, Tan J, Zhu L, Lou D, Cen X. 2017. Comparative analysis of the gut microbiota of black bears in china using high-throughput sequencing. Molecular Genetics and Genomics 292, 407-414 DOI 10.1007/s00438-016-1282-0.

Spiljar M, Merkler D, Trajkovski M. 2017. The immune system bridges the gut microbiota with systemic energy homeostasis: focus on TLRs, mucosal barrier and SCFAs. Frontiers in Immunology 8, 1353 DOI 10.3389/fimmu.2017.01353.

Thomas F, Hehemann JH, Rebuffet E, Czjzek M, Michel G. 2011. Environmental and gut bacteroidetes: the food connection. Frontiers in Microbiology 2, 93 DOI 10.3389/fmicb.2011.00093.

Thursby E, Juge N. 2017. Introduction to the human gut microbiota. Biochemical Journal 474, 1823-1836 DOI 10.1042/BCJ20160510.

Uetz P, Hošek J, Hallermann J. 2016. The Reptile Database. Available at: http://www.reptile-database.org.

Waite DW, Taylor MW. 2014. Characterizing the avian gut microbiota: membership, driving influences, and potential function. Frontiers in Microbiology 5, 223 DOI 10.3389/fmicb.2014.00223.

Wexler HM. 2007. Bacteroides: the good, the bad, and the nitty-gritty. Clinical Microbiology Reviews 20, 593-621 DOI 10.1128/CMR.00008-07.

White JR, Nagarajan N, Pop M. 2009. Statistical methods for detecting differentially abundant features in clinical metagenomic samples. PLoS Computational Biology 5, e1000352 DOI 10.1371/journal.pcbi.1000352.

Xu J, Bjursell MK, Himrod J, Deng S, Carmichael LK, Chiang HC, Hooper LV, Gordon JI. 2003. A genomic view of the human-bacteroides thetaiotaomicron symbiosis. Science 299, 2074-2076 DOI 10.1126/science.1080029. 
432 Yuan ML., Dean SH, Longo AV, Rothermel BB, Tuberville TD, Zamudio KR. 2015. Kinship, inbreeding and fine-scale spatial structure influence gut microbiota in a hindgut-fermenting tortoise. Molecular Ecology 24, 2521-2536. 


\section{Figure 1 (on next page)}

Composition of the gut microbiotas of four snake species by bacterial (A) phylum and (B) genus.

Na: Naja atra group, Pmu: Ptyas mucosus group, Ec: Elaphe carinata group, and Da: Deinagkistrodon acutus group. 
PeerJ

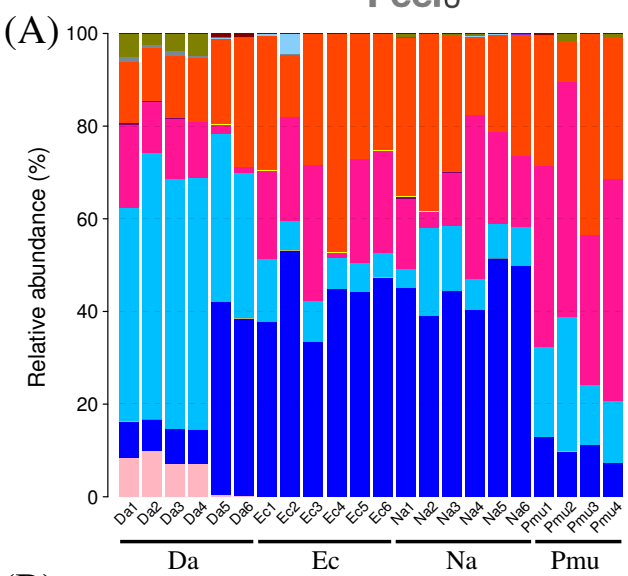

(B)

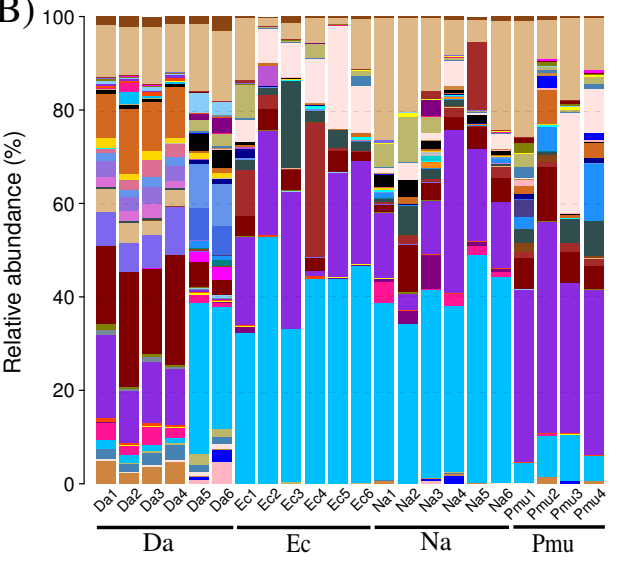

$\square$ Actinobacteria

- Bacteroidetes

$\square$ Candidatus_Saccharibacteria

$\square$ Chlamydiae

$\square$ Cyanobacteria

$\square$ Deferribacteres

$\square$ Firmicutes

$\square$ Fusobacteria

$\square$ Lentisphaerae

$\square$ Planctomycetes

$\square$ Proteobacteria

$\square$ Spirochaetes

$\square$ Synergistetes

$\square$ Tenericutes

$\square$ Verrucomicrobia

unclassified

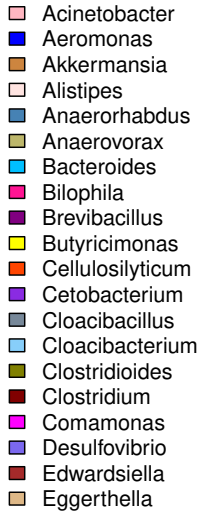

$\square$ Enterococcus

$\square$ Escherichia

$\square$ Eubacterium

$\square$ Flavobacterium

$\square$ Fusobacterium

$\square$ Gelidibacter

$\square$ Gordonibacter

$\square$ Hungatella

$\square$ Ignatzschineria

$\square$ Lachnoclostridium

$\square$ Lactobacillus

$\square$ Laribacter

- Morganella

$\square$ Mycoplasma

$\square$ Myroides

$\square$ Oceanisphaera

$\square$ Odoribacter

$\square$ Paeniclostridium

- Parabacteroides

$\square$ Paraclostridium
Peptostreptococcus

Phascolarctobacterium

$\square$ Plesiomonas

$\square$ Proteus

$\square$ Providencia

$\square$ Pseudarthrobacter

$\square$ Pseudoalteromonas

Pseudomonas

$\square$ Raoultella

$\square$ Ruminiclostridium

$\square$ Ruminococcus

$\square$ Shewanella

$\square$ Sutterella

$\square$ Terrisporobacter

- Turicibacter

$\square$ Vagococcus

$\square$ Victivallis

$\square$ Wohlfahrtiimonas

$\square$ Unclassified

$\square$ Others $(<0.5 \%)$ 


\section{Figure 2 (on next page)}

Genus-level phylogeny of gut microbiota from four snake species.

Genera are colored by phylum. 


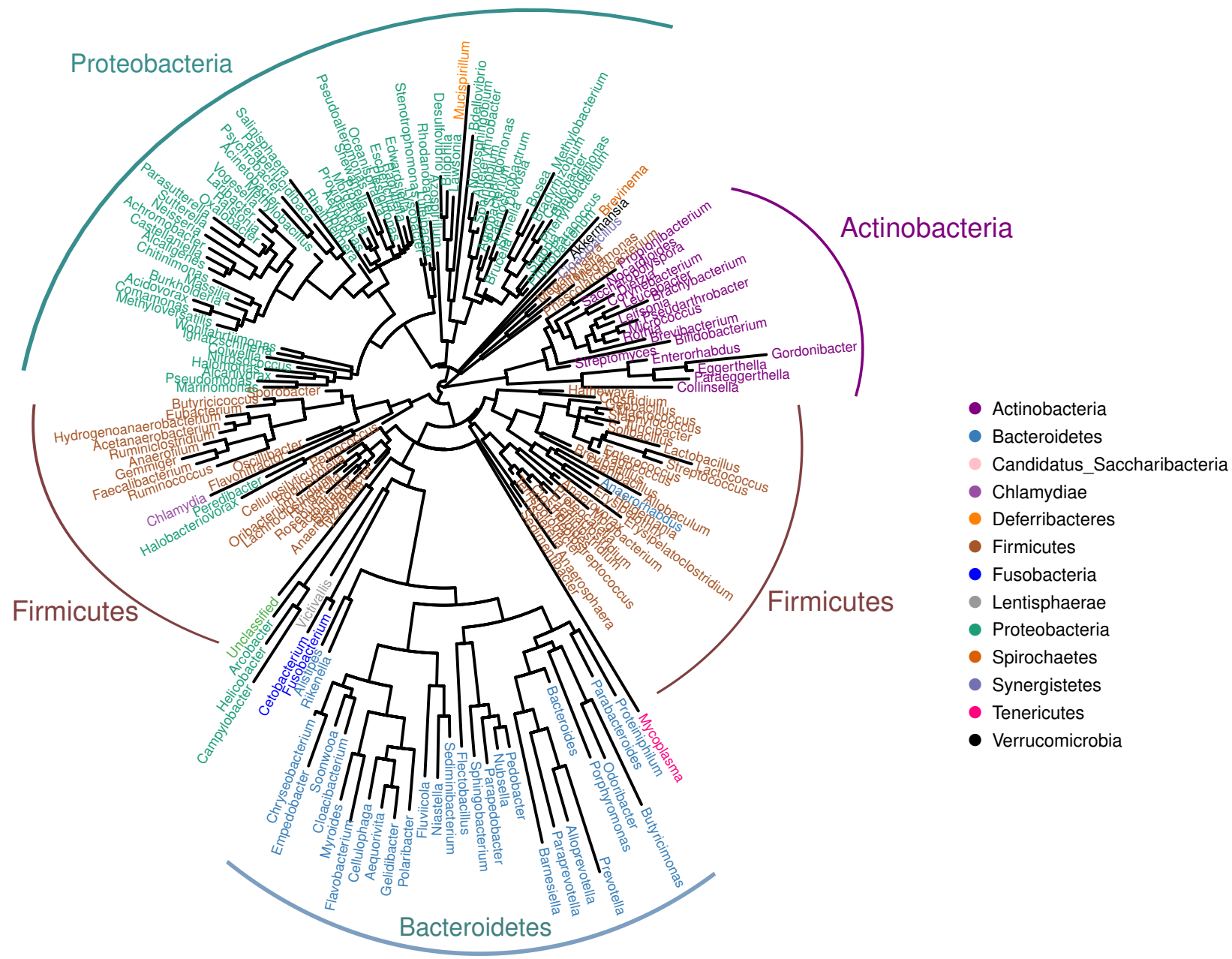


Figure 3 (on next page)

Alpha diversity, beta diversity, and principal component analysis of the bacterial communities across four snake species.

(A) Observed species (Sobs) index and (B) Shannon's diversity index. The top and bottom of each box indicate the first and third quartiles, the line inside the box indicates the median, and the ends of the dotted lines represent the minimum and the maximum. (C) Cluster tree generated based on Bray-Curtis distances. (D) The variation explained by the plotted principal component is indicated by the axis labels. Na: Naja atra group, Pmu: Ptyas mucosus group, Ec: Elaphe carinata group, and Da: Deinagkistrodon acutus group. 
PeerJ

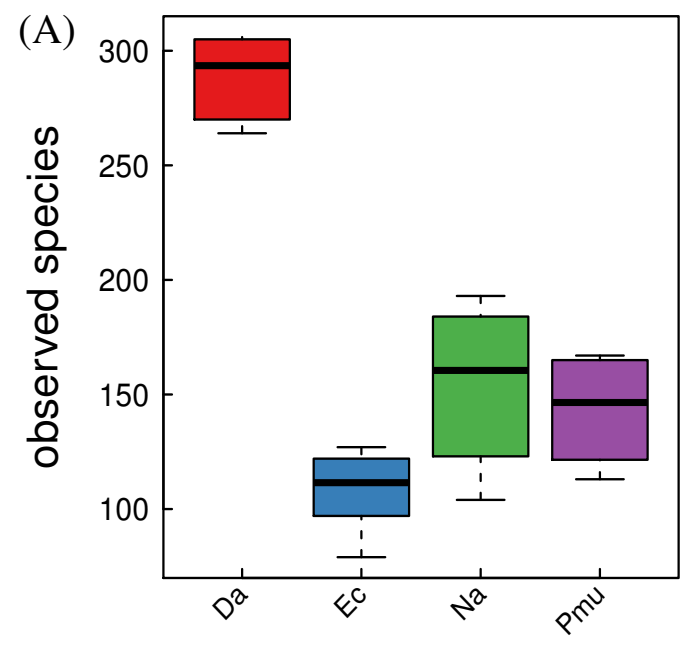

(C)

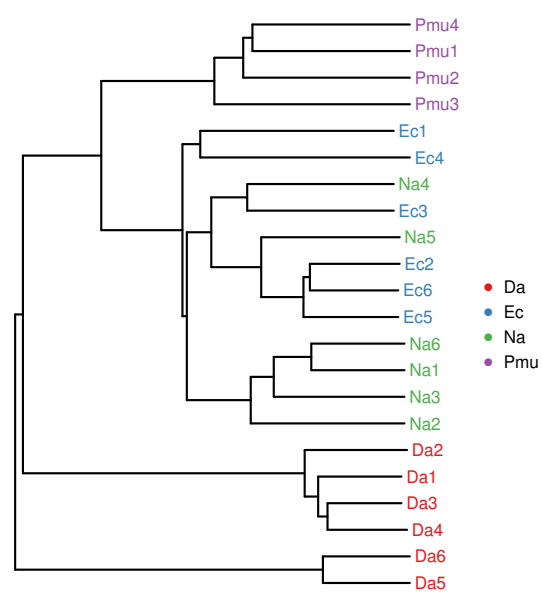

Manuscript to be reviewed

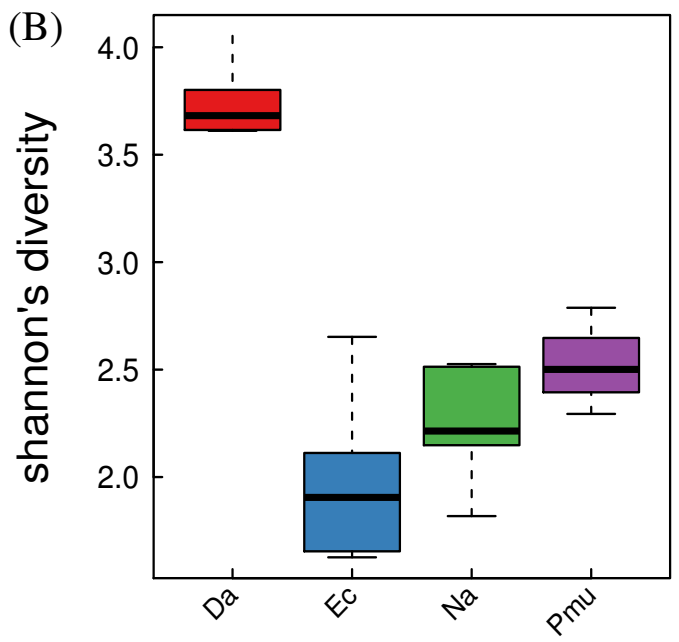

(D)

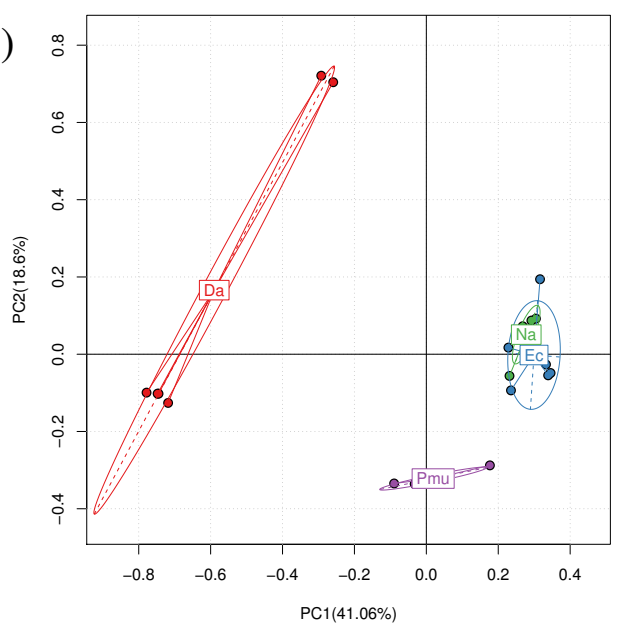




\section{Figure 4 (on next page)}

Heatmap showing the genus-level bacterial community composition in the gut microbiotas of four snake species.

Na: Naja atra group, Pmu: Ptyas mucosus group, Ec: Elaphe carinata group, and Da: Deinagkistrodon acutus group. 


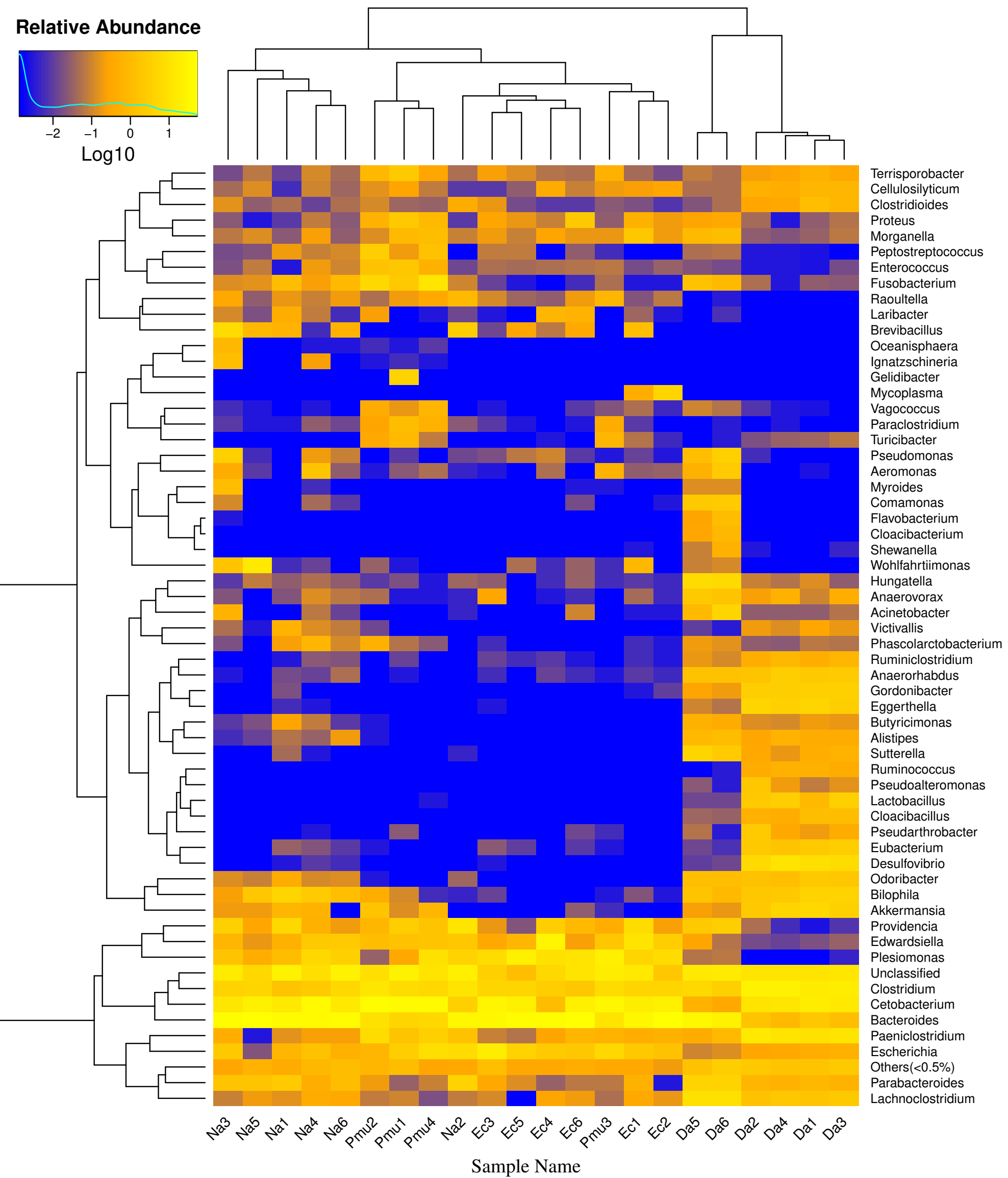


Figure $\mathbf{5}$ (on next page)

A cladogram showing the differences in relative abundance of taxa at five levels across four snake species.

The plot was generated using the online LEfSe project. The red, green, blue, and purple circles mean that four snake species showed differences in relative abundance, and yellow circles mean non-significant differences. 


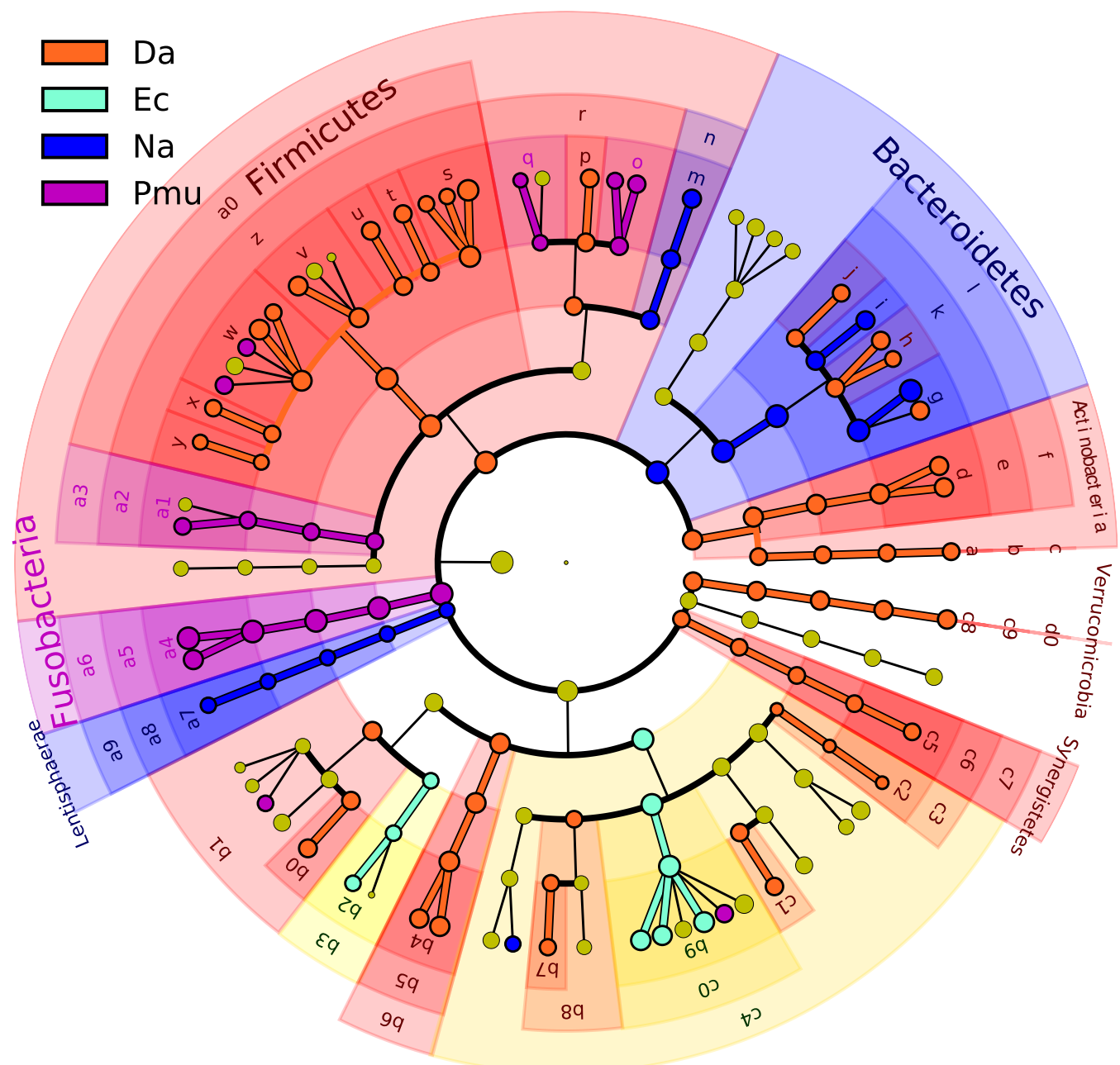

\begin{tabular}{|c|c|}
\hline$\square$ & a: Micrococcaceae \\
\hline$\square$ & b: Micrococcales \\
\hline$\square$ & c: Actinobacteria \\
\hline$\square$ & d: Eggerthellaceae \\
\hline$\square$ & e: Eggerthellales \\
\hline$\square$ & f: Coriobacteriia \\
\hline & g: Bacteroidaceae \\
\hline$\square$ & h: Odoribacteraceae \\
\hline & i: Porphyromonadaceae \\
\hline$\square$ & J j: Rikenellaceae \\
\hline & k: Bacteroidales \\
\hline & I: Bacteroidia \\
\hline & m: Paenibacillaceae \\
\hline & n: Bacillales \\
\hline コ & o: Enterococcaceae \\
\hline$\square$ & p: Lactobacillaceae \\
\hline$\square$ & q: Streptococcaceae \\
\hline$\square$ & r: Lactobacillales \\
\hline$\square$ & s: Clostridiaceae \\
\hline$\square$ & t:Clostridiales_Family_XIII_Incertae_Sedis \\
\hline$\square$ & u: Eubacteriaceae \\
\hline$\square$ & v: Lachnospiraceae \\
\hline$\square$ & w: Peptostreptococcaceae \\
\hline$\square$ & x: Ruminococcaceae \\
\hline$\square$ & y: Unclassified \\
\hline$\square$ & z: Clostridiales \\
\hline$\square$ & a0: Clostridia \\
\hline$\square$ & a1: Erysipelotrichaceae \\
\hline$\square$ & a2: Erysipelotrichales \\
\hline$\square$ & a3: Erysipelotrichia \\
\hline$\square$ & a4: Fusobacteriaceae \\
\hline$\square$ & a5: Fusobacteriales \\
\hline コ & a6: Fusobacteriia \\
\hline & a7: Victivallaceae \\
\hline & a8: Victivallales \\
\hline 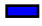 & a9: Lentisphaeria \\
\hline$\square$ & כo: Sutterellaceae \\
\hline$\square$ & J b1: Burkholderiales \\
\hline$\square$ & b2: Chromobacteriaceae \\
\hline$\square$ & b3: Neisseriales \\
\hline$\square$ & b4: Desulfovibrionaceae \\
\hline$\square$ & b5: Desulfovibrionales \\
\hline$\square$ & b6: Deltaproteobacteria \\
\hline$\square$ & b7: Pseudoalteromonadaceae \\
\hline$\square$ & b8: Alteromonadales \\
\hline$\square$ & b9: Enterobacteriaceae \\
\hline$\square$ & c0: Enterobacteriales \\
\hline$\square$ & c1: Moraxellaceae \\
\hline$\square$ & c2: Xanthomonadaceae \\
\hline$\square$ & c3: Xanthomonadales \\
\hline$\square$ & c4: Gammaproteobacteria \\
\hline$\square$ & c5: Synergistaceae \\
\hline$\square$ & c6: Synergistales \\
\hline$\square$ & c7: Synergistia \\
\hline$\square$ & c8: Akkermansiaceae \\
\hline$\square$ & c9: Verrucomicrobiales \\
\hline$\square$ & do: Verrucomicrobiae \\
\hline
\end{tabular}




\section{Table $\mathbf{1}$ (on next page)}

Composition of the fecal microbiotas of four snake species.

Na: Naja atra group, Pmu: Ptyas mucosus group, Ec: Elaphe carinata group, and Da: Deinagkistrodon acutus group. 
1

\begin{tabular}{llllll}
\hline Group & Number of Phyla & $\begin{array}{l}\text { Number of } \\
\text { classes }\end{array}$ & $\begin{array}{l}\text { Number of } \\
\text { orders }\end{array}$ & $\begin{array}{l}\text { Number of } \\
\text { families }\end{array}$ & $\begin{array}{l}\text { Number of } \\
\text { genera }\end{array}$ \\
\hline $\mathrm{Na}$ & 11 & 17 & 20 & 31 & 49 \\
$\mathrm{Pmu}$ & 11 & 16 & 19 & 28 & 44 \\
$\mathrm{Ec}$ & 9 & 15 & 19 & 27 & 44 \\
$\mathrm{Da}$ & 12 & 18 & 22 & 34 & 53 \\
Total & 15 & 18 & 22 & 35 & 58 \\
\hline
\end{tabular}

2 


\section{Table 2 (on next page)}

The differences in relative abundance $(\% \pm S D)$ of the top five most abundant phylum of four snake species.

Na: Naja atra group, Pmu: Ptyas mucosus group, Ec: Elaphe carinata group, and Da:

Deinagkistrodon acutus group. The significances of Bacteroidetes, Firmicutes, Fusobacteria, and Proteobacteria were determined using the General Linear Model, whereas the Generalized Linear Models was used to examine the significances of Actinobacteria. 


\begin{tabular}{|c|c|c|c|c|c|c|}
\hline Top five most abundant phyla & $\mathrm{Na}$ group & Pmu group & Ec group & Da group & $\mathrm{F}$ & $P$ \\
\hline Bacteroidetes & $45.07 \pm 4.92$ & $10.22 \pm 2.32$ & $43.54 \pm 6.93$ & $18.24 \pm 16.89$ & $=16.04$ & $<0.001$ \\
\hline Proteobacteria & $27.67 \pm 8.10$ & $27.74 \pm 14.28$ & $28.31 \pm 10.81$ & $16.38 \pm 6.08$ & $=2.06$ & $=0.14$ \\
\hline Fusobacteria & $16.81 \pm 10.55$ & $42.53 \pm 8.38$ & $19.42 \pm 9.59$ & $9.57 \pm 6.56$ & $=11.40$ & $<0.001$ \\
\hline Firmicutes & $9.91 \pm 5.45$ & $18.71 \pm 7.51$ & $7.88 \pm 3.04$ & $46.54 \pm 10.73$ & $=36.49$ & $<0.001$ \\
\hline Actinobacteria & $0.02 \pm 0.01$ & $0.07 \pm 0.06$ & $0.01 \pm 0.01$ & $8.35 \pm 9.93$ & $=2.10$ & $=0.15$ \\
\hline
\end{tabular}

1 